Document downloaded from:

http://hdl.handle.net/10251/98356

This paper must be cited as:

Antezana-Julian, WO.; Blas, CD.; García-Rebollar, P.; Rodríguez, C.; Beccaccia, A.; Ferrer Riera, P.; Cerisuelo, A.... (2016). Composition, potential emissions and agricultural value of pig slurry from Spanish commercial farms. Nutrient Cycling in Agroecosystems. 104(2):159173. doi:10.1007/s10705-016-9764-3

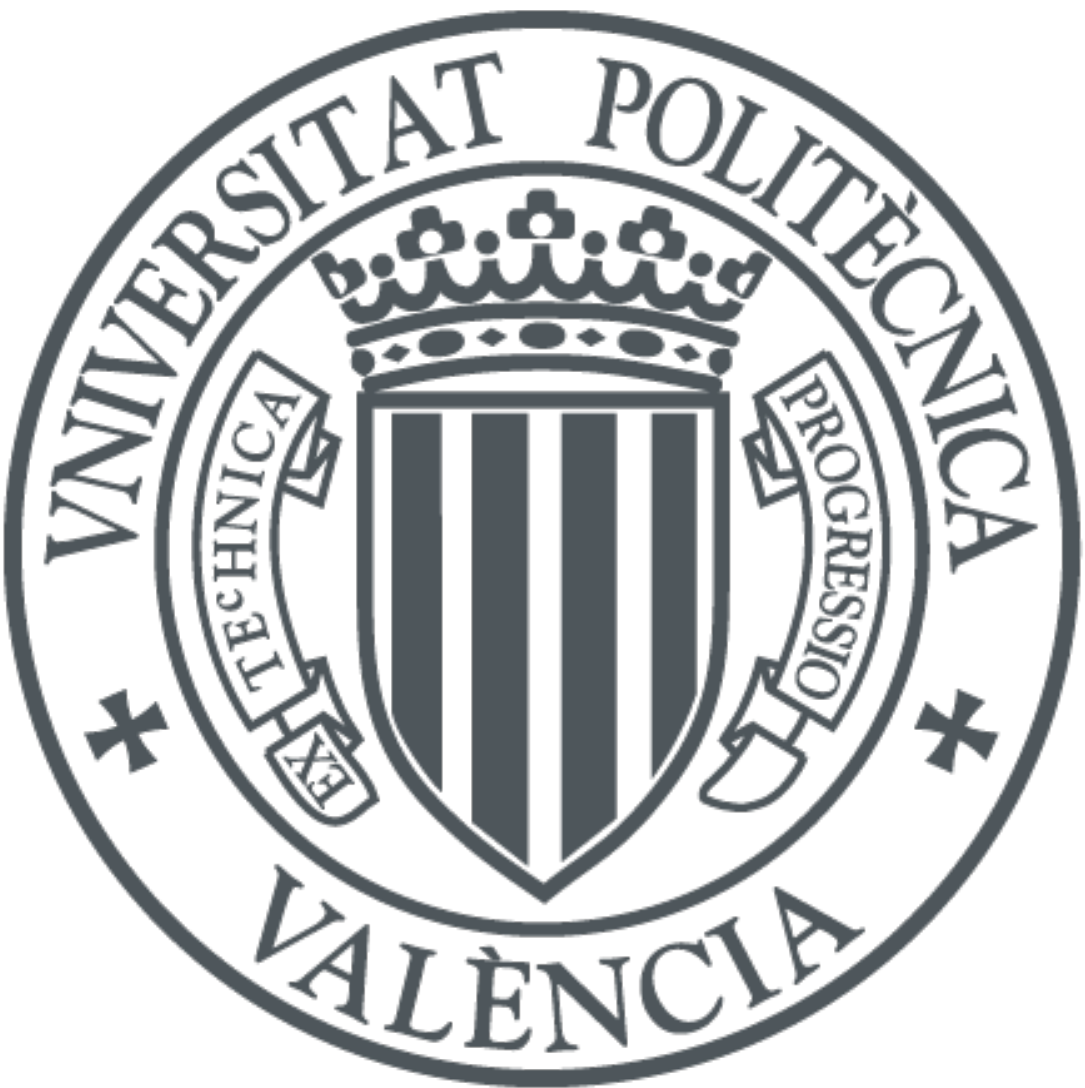

The final publication is available at

http://doi.org/10.1007/s10705-016-9764-3

Copyright Springer-Verlag

Additional Information 


\title{
Composition, potential emissions and agricultural value of pig slurry from Spanish commercial farms
}

\author{
W. Antezana - C. De Blas - P. García-Rebollar · C. Rodríguez • \\ A. Beccaccia - P. Ferrer - A. Cerisuelo - V. Moset \\ F. Estellés $\cdot$ M. Cambra-López $\cdot$ S. Calvet
}

\begin{abstract}
Pig slurry is a valuable fertilizer for crop production but at the same time its management may pose environmental risks. Slurry samples were collected from 77 commercial farms of four animal categories (gestating and lactating sows, nursery piglets and growing pigs) and analyzed for macronutrients, micronutrients, heavy metals and volatile fatty acids. Emissions of ammonia $\left(\mathrm{NH}_{3}\right)$ and biochemical methane potential (BMP) were quantified. Slurry electrical conductivity, $\mathrm{pH}$, dry matter content and ash content were also determined. Data analysis included an analysis of correlations among variables, the development of prediction models for gaseous emissions and the
\end{abstract}

analysis of nutritional content of slurries for crop production. Descriptive information is provided in this work and shows a wide range of variability in all studied variables. Animal category affected some physicochemical parameters, probably as a consequence of different slurry management and use of cleaning water. Slurries from gestating sows and growing pigs tended to be more concentrated in nutrients, whereas the slurry from lactating sows and nursery piglets tended to be more diluted. Relevant relationships were found among slurry characteristics expressed in fresh basis and gas emissions. Predictive models using on-farm measurable parameters were obtained for $\mathrm{NH}_{3}\left(\mathrm{R}^{2}=0.51\right)$ and $\mathrm{CH}_{4}$ 
$\left(\mathrm{R}^{2}=0.76\right)$, which suggests that BMP may be estimated in commercial farms from easily determined slurry characteristics. Finally, slurry nutrient composition was highly variable. Therefore, complete analyses of slurries should be performed for an effective and environmental friendly land application.

Keywords Ammonia emission - Methane emission . Fertilizer value $\cdot$ Prediction model $\cdot$ Slurry characterization

$\begin{array}{ll}\text { Abbreviations } \\ \mathrm{BMP} \text { Biochemical methane potential } \\ \mathrm{CH}_{4} & \text { Methane } \\ \mathrm{DM} & \text { Dry matter } \\ \mathrm{EC} & \text { Electrical conductivity } \\ \mathrm{CV} & \text { Coefficient of variation } \\ \mathrm{NH}_{3} & \text { Ammonia } \\ \mathrm{OM} & \text { Organic matter } \\ \mathrm{SV} & \text { Volatile solids } \\ \mathrm{TKN} & \text { Total Kjeldahl nitrogen } \\ \mathrm{TAN} & \text { Total ammonia nitrogen } \\ \text { VFA } & \text { Volatile fatty acids }\end{array}$

\section{Introduction}

At present, there is an increasing concern about the environmental impacts associated with intensive and concentrated livestock production. This is especially the case for pig production which currently has a global population of over 1 billion heads, mainly concentrated in intensive producing areas. Spain is the second largest pig producer in Europe and the sixth in the world, with about 26 million animal places (EUROSTAT 2015). Slurry management is influencing emissions of atmospheric pollutants such as ammonia $\left(\mathrm{NH}_{3}\right)$, nitrous oxide $\left(\mathrm{N}_{2} \mathrm{O}\right)$ and methane $\left(\mathrm{CH}_{4}\right)($ Hristov et al. 2013; Viguria et al. 2015) and it is also linked to potentially harmful effects for soil and water (Aarnink and Verstegen 2007).

Nevertheless, slurry should be considered a valuable resource as fertilizer because it contains significant amounts of most nutrients required by crops (Villar et al. 2004; Penha et al. 2015) plus organic substance which is important for soil fertility. The positive or negative effects of land application of slurry may depend on slurry characteristics (Villamar et al. 2013), dosing (Iguácel et al. 2011) or environmental conditions during application and hereafter (Webb et al. 2014). Moreover, pig slurry is used as a renewable source of energy due to its potential to produce $\mathrm{CH}_{4}$ under anaerobic conditions (Ferrer et al. 2014; Zhang et al. 2014).

The composition of pig slurry from commercial farms is highly variable due to differences among housing systems, feed composition, climate or farm management (Beccaccia et al. 2015; Moral et al. 2005b, 2008; Sánchez and González 2005). In recent years, several studies have been published either characterizing physicochemical composition of slurries from commercial pig farms (Suresh et al. 2009; Parera i Pous et al. 2010), or evaluating the potential to emit $\mathrm{NH}_{3}$ and greenhouse gases (GHG) form slurries obtained under experimental conditions (Antezana et al. 2015; Galassi et al. 2010; Hernández et al. 2013; Jarret et al. 2012 among others). However, there is scarce information on the potential emissions of GHG and $\mathrm{NH}_{3}$ of slurries from commercial pig farms.

Understanding the relationships between physicochemical properties of slurry can provide a basis for estimating the fertilizer value of slurry, thus facilitating its more efficient use in agriculture and reducing the potential risks to the environment (Scotford et al. 1998; Díez et al. 2006; Thygesen et al. 2012).

It is therefore necessary to evaluate in detail the physicochemical characteristics of slurries in commercial farms, to analyze their relationships with the emission of $\mathrm{CH}_{4}$ and $\mathrm{NH}_{3}$ and to examine the potential fertilizer value of slurries. A previous study (Beccaccia et al. 2015) analyzed relationships among different feed and slurry characteristics and potential gaseous emissions. The aim of this study was to analyze in depth the variability in mineral composition and gas emission of pig slurry samples from commercial pig farms, to predict potential $\mathrm{NH}_{3}$ emissions and the biochemical methane potential (BMP) in commercial farms and to explore limitations when using commercial slurries as fertilizers.

\section{Materials and methods}

Description of the selected farms and sample collection

A total of 77 samples of pig slurry from farms located in the Center and East of Spain were analyzed in order 
to account for potential variations in composition and emissions. A detailed description of the survey protocol and analysis can be found in Beccaccia et al. (2015). Samples were classified according to the animal category: 15 samples from gestating sows, 14 from lactating sows, 14 from nursery piglets and 34 from growing pigs. Table 1 summarizes the most relevant characteristics of the surveyed farms.

Sampling was designed to be representative of the slurry extracted from the pits under commercial conditions. A minimum of five aliquots (2-L) were taken during the pit discharge at equidistant time intervals. Composite samples were then thoroughly mixed in a $15 \mathrm{~L}$ container and subsamples were conditioned for the corresponding analyses. All samples were kept in sealed containers at $5{ }^{\circ} \mathrm{C}$ until laboratory processing, which was made within $24 \mathrm{~h}$.

Physicochemical analysis

All pig slurry samples were analyzed to determine dry matter (DM), organic matter (OM) and total Kjeldahl nitrogen (TKN) according to APHA (2005). Also, pH, electric conductivity (EC), total ammonia nitrogen (TAN) (4500 $\mathrm{NH}_{3}-\mathrm{B}$ and $4500 \mathrm{NH}_{3}-\mathrm{C}$ procedures)
(APHA 2005), and volatile fatty acids (VFA) (Jouany 1982) were determined. Volatile fatty acids were analyzed using a Focus Gas Chromatograph (Thermo, Milan, Italy) equipped with a split/splitless injector and a flame ionization detector with the addition of an internal standard (4-metil valeric).

Macronutrients, micronutrients and heavy metal concentrations ( $\mathrm{P}, \mathrm{K}, \mathrm{Ca}, \mathrm{Mg}, \mathrm{S}, \mathrm{Al}, \mathrm{B}, \mathrm{Cd}, \mathrm{Co}, \mathrm{Cr}, \mathrm{Cu}$, $\mathrm{Fe}, \mathrm{Li}, \mathrm{Mn}, \mathrm{Mo}, \mathrm{Na}, \mathrm{Ni}, \mathrm{Pb}$ and $\mathrm{Zn}$ ) were determined after acid digestion by inductive-optical coupled plasma spectrophotometry (ICP-OES, ICAP 6500 Duo, Thermo Scientific, Walthamm, MA, USA).

\section{Ammonia emissions}

Ammonia emissions assays were performed with an ammonia trap system similar to that described by Ndegwa et al. (2009). Slurry subsamples of $0.6 \mathrm{~kg}$ with a volume: surface ratio of $0.0952 \mathrm{~m}^{3} / \mathrm{m}^{2}$ were placed in duplicate in 1-L closed containers maintained at $25^{\circ} \mathrm{C}$ in a thermostatic water bath (Selecta, Spain). Containers were connected to an air pump which extracted air at a constant airflow rate of $1.2 \mathrm{~L} \mathrm{~min}^{-1}$. During 11 consecutive days, the air was forced to pass through two absorption flasks

Table 1 Main operation and installation characteristics of the farms surveyed

\begin{tabular}{|c|c|c|c|c|}
\hline & Gestating sows & Lactating sows & Nursery piglets & Growing pigs \\
\hline Number of farms & 15 & 14 & 14 & 34 \\
\hline Animal places per farm (average) & 770 & 162 & 2319 & 3132 \\
\hline Type of housing & Group & Individual & Group & Group \\
\hline Feeding type & Dry & $\begin{array}{l}\text { Dry }(71 \%) \\
\text { Wet }(29 \%)\end{array}$ & Dry & Dry \\
\hline Feed restriction & Yes & $\begin{array}{l}\text { No }(79 \%) \\
\text { Yes }(21 \%)\end{array}$ & No & No \\
\hline \multicolumn{5}{|l|}{ Slurry accumulation } \\
\hline$<1$ month & $21 \%$ & $93 \%$ & $50 \%$ & $41 \%$ \\
\hline $1-3$ months & $7 \%$ & $7 \%$ & $50 \%$ & $24 \%$ \\
\hline$>3$ months & $71 \%$ & $0 \%$ & $0 \%$ & $35 \%$ \\
\hline \multicolumn{5}{|l|}{ Slat } \\
\hline Partial & $64 \%$ & $21 \%$ & $21 \%$ & $41 \%$ \\
\hline Total & $36 \%$ & $79 \%$ & $79 \%$ & $59 \%$ \\
\hline \multicolumn{5}{|l|}{ Ventilation type } \\
\hline Natural & $86 \%$ & $57 \%$ & $43 \%$ & $78 \%$ \\
\hline Mechanical & $14 \%$ & $43 \%$ & $57 \%$ & $16 \%$ \\
\hline
\end{tabular}


(impingers) in serial containing $100 \mathrm{~mL}$ of sulphuric acid $0.1 \mathrm{~N}$ each. The acid solution was replaced daily during the first 5 days, and every $48 \mathrm{~h}$ until the end of the assay (day 11). The $\mathrm{NH}_{3}$ trapped in the impingers was quantified following $4500 \mathrm{NH} 3-\mathrm{D}$ procedure (APHA 2005) using a detection electrode (Orion High Performance $\mathrm{NH}_{3}$ Electrode, model 9512HPBNWP, Thermo Scientific, USA).

\section{Biochemical methane potential (BMP)}

The BMP was determined in a batch assay using $120 \mathrm{~mL}$ bottles following the methodology described by Angelidaki et al. (2009). Inoculum from a mesophilic pig slurry anaerobic digester reactor was used. The inoculum was pre-incubated during 15 days at $35^{\circ} \mathrm{C}$ in order to deplete the residual biodegradable organic material. Pig slurry and inoculum were mixed to obtain an inoculum to substrate ratio of 1 in $\mathrm{OM}$ basis. Each sample was tested out by triplicate. Additionally, three blank bottles containing degasified inoculum were included in the measurements in order to determine the inoculum endogenous $\mathrm{CH}_{4}$ production. The endogenous $\mathrm{CH}_{4}$ production from the inoculum was subtracted from the $\mathrm{CH}_{4}$ produced by the pig slurry on each biogas sampling day. After filling, each bottle was sealed with butyl rubber stoppers and aluminum crimps and the headspace was flushed with pure $\mathrm{N}_{2}$ for $2 \mathrm{~min}$. Bottles were then incubated at $35^{\circ} \pm 1{ }^{\circ} \mathrm{C}$ for 100 days. During incubation, biogas volume in each bottle was regularly monitored (from 1 to 10 days depending on biogas production) by pressure measurement of the headspace using a manometer (Delta Ohm, HD 9220, Italy). Methane concentration in the biogas was further analyzed using a Focus Gas Chromatograph (Thermo, Milan, Italy) equipped with a split/splitless injector and a flame ionization detector. Finally, the BMP was expressed per volume of slurry considering the OM content.

\section{Statistical analyses}

All slurry components were expressed and analyzed in wet basis. The descriptive statistical analysis of the results of the variables in this study was made by animal category through PROC MEANS of SAS Institute (2008). The effect of the animal type in the studied variables was analyzed using PROC GLM and
PROC T TEST of SAS Institute (2008). The Correlation analysis among physicochemical slurry characteristics, as well as $\mathrm{NH}_{3}$ and BMP, was done using PROC CORR of SAS Institute (2008). A multiple regression analysis was also conducted with a stepwise variable selection process using the PROC REG of SAS Institute (2008) to establish prediction models of potential emissions from easily measurable slurry characteristics (i.e. EC, $\mathrm{pH}$ and DM).

From the results of the physicochemical characterization of slurry, potential scenarios of nutrient loads associated to land application were obtained. These scenarios corresponded to limitations of organic manure application according to international regulations, in particular the European Union Directive 91/676/CE which specifies that the livestock manure applied to land each year shall not exceed the amount containing $170 \mathrm{~kg} \mathrm{~N} \mathrm{ha}^{-1}$.

\section{Results and discussion}

Physicochemical slurry characterization

Summary statistics for the physicochemical characterization of slurry for the different animal categories are presented in Table 2 . As shown in this table, slurry characteristics varied considerably among and within animal categories. Only for some characteristics the category of animal was found to have a statistically significant effect. Correlations among the main physicochemical characteristics are shown in Table 3.

The main results of this study are in agreement with those reported for pig slurries from commercial pig farms in Spain (Moral et al. 2005a, b; Parera i Pous et al. 2010; Sánchez and González 2005, among others) and other countries (Abubaker et al. 2015; Martínez-Suller et al. 2008; Olusegun 2014; Suresh et al. 2009; Villamar et al. 2012). The EC in pig slurry ranged from 2.65 to $53.46 \mathrm{mS} \mathrm{cm}{ }^{-1}$, and was higher for growing pigs and gestating sows than for lactating sows and nursery piglets $(p<0.05)$. This difference may be attributed to the housing system and management practices such as slurry removal frequency and use of cleaning water. In fact, animal categories showing higher EC are those in which slurries tend to be stored for a longer time in slurry pits (see Table 1), thus enhancing drying and mineralization of slurry. On the contrary, lactating sows and weaners are those with 
Table 2 Physicochemical characterization of pig slurries by different animal categories

\begin{tabular}{|c|c|c|c|c|c|c|c|c|c|c|}
\hline \multirow[t]{2}{*}{ Parameter } & \multicolumn{2}{|c|}{$\begin{array}{l}\text { All samples } \\
(\mathrm{n}=77)\end{array}$} & \multicolumn{4}{|c|}{ Growing pigs $(\mathrm{n}=34)$} & \multicolumn{4}{|c|}{ Gestating sows $(n=15)$} \\
\hline & Mean & $\mathrm{CV}$ & Mean & Min & Max & $\mathrm{CV}$ & Mean & Min & Max & $\mathrm{CV}$ \\
\hline \multicolumn{11}{|c|}{ Physicochemical characteristics } \\
\hline $\begin{array}{l}\text { Electrical conductivity } \\
\quad\left(\mathrm{mS} \mathrm{cm}^{-1}\right)\end{array}$ & 21.8 & 53.1 & $25.7 \mathrm{a}$ & 6.59 & 53.5 & 52.1 & $21.6 \mathrm{a}$ & 10.15 & 44.6 & 37.2 \\
\hline $\mathrm{pH}$ & 7.52 & 5.50 & $7.39 \mathrm{a}$ & 6.41 & 8.05 & 5.70 & $7.78 \mathrm{~b}$ & 7.54 & 8.03 & 1.8 \\
\hline Dry matter $\left(\mathrm{kg} \mathrm{m}^{-3}\right)$ & 49.3 & 78.9 & 57.09 & 7.50 & 176.7 & 71.7 & 56.05 & 4.9 & 155.4 & 92.4 \\
\hline Organic matter $\left(\mathrm{kg} \mathrm{m}^{-3}\right)$ & 36.7 & 82.8 & 43.51 & 4.50 & 145.1 & 75.8 & 41.1 & 2.8 & 120.5 & 94.9 \\
\hline $\operatorname{Ash}\left(\mathrm{kg} \mathrm{m}^{-3}\right)$ & 12.5 & 70.7 & 13.57 & 3.00 & 34.6 & 62.2 & 14.94 & 2.12 & 45.6 & 87.7 \\
\hline \multicolumn{11}{|l|}{ Macronutrients } \\
\hline $\mathrm{N}\left(\mathrm{kg} \mathrm{m}^{-3}\right)$ & 4.79 & 64.5 & $6.01 \mathrm{a}$ & 1.43 & 15.1 & 60.0 & $4.36 \mathrm{a}$ & 1.62 & 9.09 & 53.9 \\
\hline $\mathrm{P}\left(\mathrm{kg} \mathrm{m}^{-3}\right)$ & 0.860 & 90.9 & 0.831 & 0.104 & 2.09 & 63.3 & 1.31 & 0.060 & 4.58 & 105.9 \\
\hline $\mathrm{K}\left(\mathrm{kg} \mathrm{m}^{-3}\right)$ & 1.66 & 64.3 & $2.05 \mathrm{a}$ & 0.504 & 4.62 & 60.5 & $1.53 \mathrm{a}$ & 0.261 & 3.81 & 59.2 \\
\hline $\mathrm{Ca}\left(\mathrm{kg} \mathrm{m}^{-3}\right)$ & 1.23 & 95.9 & $1.13 \mathrm{a}$ & 0.227 & 3.14 & 71.9 & $1.97 \mathrm{~b}$ & 0.115 & 6.45 & 107.9 \\
\hline $\operatorname{Mg}\left(\mathrm{kg} \mathrm{m}^{-3}\right)$ & 0.560 & 84.1 & 0.59 & 0.081 & 1.39 & 61.8 & 0.738 & 0.034 & 2.66 & 107.0 \\
\hline $\mathrm{S}\left(\mathrm{kg} \mathrm{m}^{-3}\right)$ & 0.300 & 74.7 & 0.34 & 0.056 & 0.953 & 71.5 & 0.310 & 0.043 & 0.849 & 86.7 \\
\hline \multicolumn{11}{|c|}{ Micronutrients and heavy metals } \\
\hline $\mathrm{Al}\left(\mathrm{g} \mathrm{m}^{-3}\right)$ & 43.3 & 85.2 & 41.57 & 3.20 & 115.9 & 78.1 & 47.9 & 1.44 & 152.9 & 99.5 \\
\hline B $\left(\mathrm{g} \mathrm{m}^{-3}\right)$ & 1.84 & 69.5 & 2.13 & 0.483 & 6.48 & 70.4 & 1.55 & 0.185 & 3.73 & 73.0 \\
\hline $\mathrm{Cd}\left(\mathrm{g} \mathrm{m}^{-3}\right)$ & 0.013 & 88.3 & 0.011 & 0.002 & 0.034 & 78.8 & 0.015 & 0.000 & 0.049 & 103.8 \\
\hline $\operatorname{Co}\left(\mathrm{g} \mathrm{m}^{-3}\right)$ & 0.686 & 317.2 & 0.507 & 0.014 & 5.70 & 208 & 0.840 & 0.018 & 8.9 & 268.2 \\
\hline $\mathrm{Cr}\left(\mathrm{g} \mathrm{m}^{-3}\right)$ & 0.602 & 112.0 & 0.61 & 0.031 & 1.95 & 87.1 & 0.880 & 0.018 & 4.36 & 133.1 \\
\hline $\mathrm{Cu}\left(\mathrm{g} \mathrm{m}^{-3}\right)$ & 12.7 & 126.3 & $10.4 \mathrm{a}$ & 0.684 & 59.75 & 113 & $6.12 \mathrm{a}$ & 0.236 & 17.6 & 92.2 \\
\hline $\mathrm{Fe}\left(\mathrm{g} \mathrm{m}^{-3}\right)$ & 85.0 & 93.1 & 73.78 & 5.95 & 189.6 & 64.1 & 90.52 & 2.92 & 302.2 & 101.7 \\
\hline $\operatorname{Li}\left(\mathrm{g} \mathrm{m}^{-3}\right)$ & 0.149 & 117.9 & 0.145 & 0.017 & 0.797 & 126 & 0.189 & 0.009 & 0.963 & 131.7 \\
\hline $\operatorname{Mn}\left(\mathrm{g} \mathrm{m}^{-3}\right)$ & 22.6 & 89.2 & 24.41 & 3.05 & 79.5 & 67.5 & 28.59 & 1.19 & 123.3 & 116.2 \\
\hline Mo $\left(\mathrm{g} \mathrm{m}^{-3}\right)$ & 0.313 & 87.5 & 0.355 & 0.044 & 1.21 & 85.1 & 0.253 & 0.008 & 0.901 & 98.3 \\
\hline $\mathrm{Na}\left(\mathrm{kg} \mathrm{m} \mathrm{m}^{-3}\right)$ & 0.425 & 69.5 & $0.527 \mathrm{a}$ & 0.108 & 1.41 & 68.2 & $0.382 \mathrm{a}$ & 0.061 & 0.912 & 58.3 \\
\hline $\mathrm{Ni}\left(\mathrm{g} \mathrm{m}^{-3}\right)$ & 0.433 & 98.2 & 0.474 & 0.047 & 1.46 & 77.3 & 0.558 & 0.015 & 2.69 & 125.6 \\
\hline $\mathrm{Pb}\left(\mathrm{g} \mathrm{m}^{-3}\right)$ & 0.128 & 111.9 & 0.096 & 0.007 & 0.335 & 78.6 & 0.115 & 0.002 & 0.316 & 91.7 \\
\hline $\mathrm{Zn}\left(\mathrm{g} \mathrm{m}^{-3}\right)$ & 109.9 & 148.1 & $74.33 \mathrm{a}$ & 3.98 & 442.8 & 123 & $45.92 \mathrm{a}$ & 1.71 & 124.9 & 86.1 \\
\hline \multicolumn{11}{|l|}{ Other characteristics } \\
\hline $\mathrm{C}\left(\mathrm{kg} \mathrm{m}^{-3}\right)$ & 20.2 & 81.3 & 24.5 & 2.52 & 77.0 & 74.9 & 21.06 & 1.35 & 56.9 & 92.1 \\
\hline TAN $\left(\mathrm{kg} \mathrm{m}^{-3}\right)$ & 3.07 & 64.6 & $3.94 \mathrm{a}$ & 0.673 & 10.3 & 59.3 & $2.87 \mathrm{ab}$ & 1.29 & 4.68 & 37.5 \\
\hline Organic $N\left(\mathrm{~kg} \mathrm{~m}^{-3}\right)$ & 1.67 & 87.9 & 2.03 & 0.176 & 7.87 & 87.1 & 1.62 & 0.141 & 5.1 & 91.9 \\
\hline VFA $\left(\mathrm{mol} \mathrm{m}^{-3}\right)$ & 75.4 & 106.1 & $105.8 \mathrm{a}$ & 0.867 & 329.7 & 87.7 & $37.4 b$ & 4.21 & 84.5 & 86.7 \\
\hline Acetic $\left(\mathrm{mol} \mathrm{m}^{-3}\right)$ & 47.2 & 112.3 & $66.55 \mathrm{a}$ & 0.618 & 238.4 & 93.7 & $28.8 \mathrm{~b}$ & 3.65 & 65.6 & 80.5 \\
\hline Propionic $\left(\mathrm{mol} \mathrm{m}^{-3}\right)$ & 14.9 & 100.8 & $19.87 \mathrm{a}$ & 0.114 & 69.4 & 82.2 & $5.85 b$ & 0.231 & 14.0 & 105.3 \\
\hline Butyric $\left(\mathrm{mol} \mathrm{m} \mathrm{m}^{-3}\right)$ & 6.49 & 164.9 & $10.36 \mathrm{a}$ & 0.014 & 47.4 & 128 & $0.750 \mathrm{~b}$ & 0.024 & 3.37 & 146.0 \\
\hline Ratio acetic/propionic & 6.42 & 117.6 & 5.40 & 0.921 & 45.2 & 151 & 8.45 & 3.59 & 16.2 & 56.6 \\
\hline Ratio acetic/butyric & 53.7 & 134.8 & $28.8 \mathrm{a}$ & 1.85 & 118.5 & 122 & $121.1 \mathrm{~b}$ & 15.6 & 325.9 & 89.4 \\
\hline Ratio butyric/propionic & 0.353 & 96.2 & 0.474 & 0.010 & 1.28 & 84 & 0.153 & 0.023 & 0.663 & 124.8 \\
\hline Ratio $\mathrm{C} / \mathrm{N}$ & 4.05 & 53.9 & 4.04 & 1.24 & 11.4 & 55.4 & 4.17 & 0.733 & 10.5 & 68.9 \\
\hline Ratio N/P & 7.82 & 61.8 & 8.65 & 2.55 & 26.1 & 49 & 7.52 & 1.41 & 30.5 & 97.3 \\
\hline Ratio N/K & 3.15 & 36.8 & 3.18 & 1.25 & 5.67 & 31.1 & 3.23 & 1.61 & 6.2 & 39.4 \\
\hline
\end{tabular}


Table 2 continued

\begin{tabular}{|c|c|c|c|c|c|c|c|c|c|}
\hline \multirow[t]{2}{*}{ Parameter } & \multicolumn{2}{|c|}{$\begin{array}{l}\text { All samples } \\
(\mathrm{n}=77)\end{array}$} & \multicolumn{3}{|c|}{ Growing pigs $(\mathrm{n}=34)$} & \multicolumn{4}{|c|}{ Gestating sows $(\mathrm{n}=15)$} \\
\hline & Mean & & Mean & Min & $\mathrm{CV}$ & Mean & Min & $\operatorname{Max}$ & $\mathrm{CV}$ \\
\hline \multicolumn{10}{|l|}{ Potential gas emission } \\
\hline Emission $\mathrm{NH}_{3}\left(\mathrm{~g} \mathrm{~m}^{-2}\right.$ day $\left.^{-1}\right)$ & 10.0 & 4.77 & $11.6 \mathrm{a}$ & 3.53 & 42 & \multicolumn{2}{|c|}{$9.37 \mathrm{a}$} & 20.0 & 41.2 \\
\hline Emission $\mathrm{CH}_{4}\left(\mathrm{~m}^{3} \mathrm{~m}^{-3}\right)$ & 9.73 & & $14.4 \mathrm{a}$ & 0.421 & 97.7 & \multicolumn{2}{|c|}{$4.09 \mathrm{~b}$} & 10.8 & 103.5 \\
\hline \multirow[t]{2}{*}{ Parameter } & \multicolumn{4}{|c|}{ Lactating sows $(\mathrm{n}=14)$} & \multicolumn{4}{|c|}{ Nursery piglets $(\mathrm{n}=14$ ) } & \multirow[t]{2}{*}{$p$ value* } \\
\hline & Mean & Min & $\operatorname{Max}$ & $\mathrm{CV}$ & Mean & Min & $\operatorname{Max}$ & $\mathrm{CV}$ & \\
\hline \multicolumn{10}{|l|}{ Physicochemical characteristics } \\
\hline Electrical conductivity $\left(\mathrm{mS} \mathrm{cm}^{-1}\right)$ & $15.5 b$ & 6.26 & 25.6 & 38.5 & $19.0 \mathrm{~b}$ & 2.65 & 46.6 & 61.5 & 0.030 \\
\hline $\mathrm{pH}$ & $7.66 \mathrm{c}$ & 7.05 & 8.16 & 3.60 & $7.43 \mathrm{ac}$ & 6.34 & 7.92 & 7.50 & 0.009 \\
\hline Dry matter $\left(\mathrm{kg} \mathrm{m}^{-3}\right)$ & 35.6 & 8.6 & 75.8 & 63.1 & 36.83 & 5.70 & 97.3 & 67.2 & 0.172 \\
\hline Organic matter $\left(\mathrm{kg} \mathrm{m}^{-3}\right)$ & 25.9 & 4.9 & 55.4 & 66.3 & 26.74 & 3.60 & 72.4 & 70.7 & 0.152 \\
\hline Ash $\left(\mathrm{kg} \mathrm{m}^{-3}\right)$ & 9.72 & 3.72 & 20.4 & 56.3 & 10.09 & 1.88 & 24.9 & 59.9 & 0.256 \\
\hline \multicolumn{10}{|l|}{ Macronutrients } \\
\hline $\mathrm{N}\left(\mathrm{kg} \mathrm{m}^{-3}\right)$ & $3.37 \mathrm{~b}$ & 1.69 & 5.97 & 39.0 & $3.71 b$ & 0.720 & 11.7 & 74.4 & 0.014 \\
\hline $\mathrm{P}\left(\mathrm{kg} \mathrm{m}^{-3}\right)$ & 0.704 & 0.107 & 1.86 & 80.7 & 0.623 & 0.111 & 1.59 & 68.3 & 0.076 \\
\hline $\mathrm{K}\left(\mathrm{kg} \mathrm{m}^{-3}\right)$ & $1.07 \mathrm{~b}$ & 0.583 & 1.58 & 32.4 & $1.42 \mathrm{a}$ & 0.216 & 3.85 & 65.7 & 0.017 \\
\hline $\mathrm{Ca}\left(\mathrm{kg} \mathrm{m}^{-3}\right)$ & $1.01 \mathrm{a}$ & 0.198 & 1.96 & 61.2 & $0.895 \mathrm{a}$ & 0.149 & 2.22 & 66.7 & 0.047 \\
\hline $\operatorname{Mg}\left(\mathrm{kg} \mathrm{m}^{-3}\right)$ & 0.477 & 0.056 & 1.28 & 80.6 & 0.386 & 0.082 & 0.856 & 63.2 & 0.193 \\
\hline $\mathrm{S}\left(\mathrm{kg} \mathrm{m}^{-3}\right)$ & 0.212 & 0.076 & 0.47 & 63.1 & 0.313 & 0.061 & 0.856 & 69.8 & 0.390 \\
\hline \multicolumn{10}{|l|}{ Micronutrients and heavy metals } \\
\hline $\mathrm{Al}\left(\mathrm{g} \mathrm{m}^{-3}\right)$ & 53.5 & 5.59 & 147.2 & 82.9 & 32.7 & 2.02 & 88.1 & 75.9 & 0.479 \\
\hline $\mathbf{B}\left(\mathrm{g} \mathrm{m}^{-3}\right)$ & 1.43 & 0.408 & 2.82 & 56.4 & 1.86 & 0.252 & 4.33 & 62.4 & 0.272 \\
\hline $\mathrm{Cd}\left(\mathrm{g} \mathrm{m}^{-3}\right)$ & 0.013 & 0.001 & 0.039 & 96.1 & 0.016 & 0.001 & 0.043 & 76.0 & 0.520 \\
\hline $\operatorname{Co}\left(\mathrm{g} \mathrm{m}^{-3}\right)$ & 1.37 & 0.019 & 16.3 & 314 & 0.273 & 0.023 & 1.16 & 129 & 0.543 \\
\hline $\mathrm{Cr}\left(\mathrm{g} \mathrm{m}^{-3}\right)$ & 0.451 & 0.037 & 1.3 & 90.2 & 0.44 & 0.042 & 1.68 & 93.0 & 0.262 \\
\hline $\mathrm{Cu}\left(\mathrm{g} \mathrm{m}^{-3}\right)$ & $5.3 \mathrm{a}$ & 0.598 & 17.2 & 93.8 & $32.8 \mathrm{~b}$ & 3.33 & 80.6 & 70.7 & 0.000 \\
\hline $\mathrm{Fe}\left(\mathrm{g} \mathrm{m}^{-3}\right)$ & 107.5 & 6.47 & 413.5 & 113 & 83.9 & 5.48 & 242.9 & 92.9 & 0.603 \\
\hline $\mathrm{Li}\left(\mathrm{g} \mathrm{m}^{-3}\right)$ & 0.145 & 0.025 & 0.434 & 88.0 & 0.118 & 0.015 & 0.305 & 82.3 & 0.755 \\
\hline $\operatorname{Mn}\left(\mathrm{g} \mathrm{m}^{-3}\right)$ & 15.8 & 2.12 & 43.4 & 82.7 & 18.3 & 2.2 & 47.9 & 76.4 & 0.280 \\
\hline Mo $\left(\mathrm{g} \mathrm{m}^{-3}\right)$ & 0.271 & 0.026 & 0.988 & 103 & 0.318 & 0.039 & 0.701 & 71.7 & 0.607 \\
\hline $\mathrm{Na}\left(\mathrm{kg} \mathrm{m}^{-3}\right)$ & $0.304 \mathrm{~b}$ & 0.127 & 0.539 & 43.6 & $0.344 \mathrm{c}$ & 0.062 & 0.826 & 70.0 & 0.047 \\
\hline $\mathrm{Ni}\left(\mathrm{g} \mathrm{m}^{-3}\right)$ & 0.303 & 0.051 & 0.732 & 71.0 & 0.331 & 0.031 & 1.03 & 90.3 & 0.298 \\
\hline $\mathrm{Pb}\left(\mathrm{g} \mathrm{m}^{-3}\right)$ & 0.181 & 0.009 & 0.691 & 105 & 0.167 & 0.017 & 0.893 & 134 & 0.187 \\
\hline $\mathrm{Zn}\left(\mathrm{g} \mathrm{m}^{-3}\right)$ & $57.9 \mathrm{a}$ & 5.12 & 189.4 & 101 & $316.8 \mathrm{~b}$ & 35.5 & 907.1 & 84.5 & 0.000 \\
\hline \multicolumn{10}{|l|}{ Other characteristics } \\
\hline $\mathrm{C}\left(\mathrm{kg} \mathrm{m}^{-3}\right)$ & 13.8 & 2.45 & 28.1 & 66.2 & 15.4 & 2.03 & 43.1 & 70.5 & 0.125 \\
\hline TAN $\left(\mathrm{kg} \mathrm{m}^{-3}\right)$ & $1.94 \mathrm{c}$ & 0.878 & 3.38 & 36.5 & $2.32 b$ & 0.325 & 7.58 & 80.2 & 0.003 \\
\hline Organic $\mathrm{N}\left(\mathrm{kg} \mathrm{m}^{-3}\right)$ & 1.22 & 0.213 & 2.4 & 58.3 & 1.32 & 0.335 & 4.44 & 79.7 & 0.241 \\
\hline VFA $\left(\mathrm{mol} \mathrm{m} \mathrm{m}^{-3}\right)$ & $30.9 b$ & 1.589 & 107.5 & 102.0 & $72.7 \mathrm{a}$ & 9.77 & 250.0 & 107 & 0.019 \\
\hline Acetic $\left(\mathrm{mol} \mathrm{m}^{-3}\right)$ & $17.7 \mathrm{~b}$ & 1.45 & 31.7 & 64.0 & $40.3 \mathrm{ab}$ & 1.83 & 165.2 & 135 & 0.038 \\
\hline Propionic $\left(\mathrm{mol} \mathrm{m}^{-3}\right)$ & $6.95 \mathrm{c}$ & 0.057 & 36.9 & 161 & $18.4 \mathrm{a}$ & 2.06 & 40.0 & 79.9 & 0.015 \\
\hline Butyric $\left(\mathrm{mol} \mathrm{m}^{-3}\right)$ & $2.30 \mathrm{~b}$ & 0.028 & 14.9 & 205 & $5.47 \mathrm{ab}$ & 0.042 & 23.8 & 148 & 0.038 \\
\hline Ratio acetic/propionic & 10.1 & 0.758 & 25.7 & 93.4 & 3.28 & 0.131 & 11.8 & 113 & 0.162 \\
\hline
\end{tabular}


Table 2 continued

\begin{tabular}{|c|c|c|c|c|c|c|c|c|c|}
\hline \multirow[t]{2}{*}{ Parameter } & \multicolumn{4}{|c|}{ Lactating sows $(\mathrm{n}=14)$} & \multicolumn{4}{|c|}{ Nursery piglets $(n=14)$} & \multirow[t]{2}{*}{$p$ value* } \\
\hline & Mean & Min & Max & $\mathrm{CV}$ & Mean & Min & Max & $\mathrm{CV}$ & \\
\hline Ratio acetic/butyric & $75.5 b$ & 1.87 & 293.7 & 114 & $32.0 \mathrm{a}$ & 2.92 & 142.9 & 141 & 0.002 \\
\hline Ratio butyric/propionic & 0.274 & 0.02 & 0.511 & 74.7 & 0.284 & 0.003 & 0.645 & 92.4 & 0.073 \\
\hline Ratio $\mathrm{C} / \mathrm{N}$ & 3.81 & 1.15 & 6.6 & 44.4 & 4.18 & 1.81 & 7.33 & 43 & 0.958 \\
\hline Ratio N/P & 7.13 & 2.58 & 20.2 & 64.9 & 6.79 & 3.04 & 12.6 & 41.6 & 0.547 \\
\hline Ratio N/K & 3.34 & 1.75 & 6.59 & 41.4 & 2.81 & 1.65 & 6.53 & 44.2 & 0.215 \\
\hline \multicolumn{10}{|l|}{ Potential gas emission } \\
\hline Emission $\mathrm{NH}_{3}\left(\mathrm{~g} \mathrm{~m}^{-2}\right.$ day $\left.^{-1}\right)$ & $7.29 \mathrm{~b}$ & 3.32 & 12.8 & 34.6 & $8.43 \mathrm{a}$ & 1.47 & 14.8 & 51.6 & 0.050 \\
\hline Emission $\mathrm{CH}_{4}\left(\mathrm{~m}^{3} \mathrm{~m}^{-3}\right)$ & $4.45 b$ & 0.223 & 10.9 & 101 & $7.33 \mathrm{a}$ & 0.822 & 25.1 & 107 & 0.019 \\
\hline
\end{tabular}

Means within the same row followed by different letters were significantly different

* ANOVA, test for comparing the animal type (growing pigs, gestating sows, lactating sows and nursery piglets)

shorter slurry accumulation time and tend to use higher volumes of cleaning water. Therefore, slurries from lactating sows and weaners tend to be more diluted, with lower DM contents. Differences in the concentration of protein and minerals in the diet of pigs among animal types (Moral et al. 2008; Beccaccia et al. 2015) may also be contributing to differences in EC. As shown in Table 3 and evidenced by previous studies (Provolo and Martínez-Suller 2007; Yagüe et al. 2012), both DM and EC are significantly correlated with most slurry characteristics. These relationships support the idea of dilution as a main factor affecting nutrient composition of slurries, and thus they have been used to propose prediction models for nutrients (Martínez-Suller et al. 2008). The $\mathrm{pH}$ ranged from 6.3 to 8.0 with low variation among animal categories. Slurry $\mathrm{pH}$ from gestating sows was on average 7.78 and it was higher than in slurries from other animal categories $(p<0.01)$. This value could probably be associated to lower concentrations of VFA (Table 2).

Regarding macronutrients (N, P, K, Ca, Mg and S) their concentration in the slurry tends to be higher for growing pigs and gestating sows, which is statistically significant in the case of $\mathrm{N}, \mathrm{K}$ and $\mathrm{Ca}$. In this regard, Sánchez and González (2005) and Moral et al. (2005a) also found higher $\mathrm{N}$ concentrations in slurries from growing pigs and gestating sows. It must be considered that nutrient excretion is a consequence of the inefficiency in their use by the animals and therefore it is affected by nutritional factors (Morazán et al. 2015; Bai et al. 2014; Patience et al. 2015), which may differ among animal categories.
A major part of the slurry nitrogen is in inorganic form, mainly as TAN, which represented $65 \%$ of the total nitrogen. These values are similar to those obtained by previous studies (Sánchez and González 2005), which reported values of $57 \%$ of TAN. As shown in Fig. 1, both $\mathrm{N}$ and TAN were positively correlated with EC $\left(\mathrm{R}^{2}=0.65\right.$ and 0.79 , respectively), which supports the conclusions of MartínezSuller et al. (2008) and Moral et al. (2005b) that these components can be predicted in practice.

In relation to the $\mathrm{P}$ content in the slurry, a wide variation was found among animal categories (ranging from 0.06 to $4.58 \mathrm{~kg} \mathrm{~m}^{-3}$ ). The average content was $0.86 \mathrm{~kg} \mathrm{~m}^{-3}$, but no statistical differences were found among animal categories. In the case of $\mathrm{K}$, the slurry from lactating sows $\left(1.07 \mathrm{~kg} \mathrm{~m}^{-3}\right)$ had a lower content than for the other categories $(p<0.05)$. Regarding the $\mathrm{Ca}$ content, the slurry from gestating sows (on average $1.97 \mathrm{~kg} \mathrm{~m}^{-3}$ ) had a higher content than for the other categories $(p<0.05)$. Finally in the case of $\mathrm{Mg}$ the average content was $0.56 \mathrm{~kg} \mathrm{~m}^{-3}$, but no statistical differences were found among animal categories. This is in accordance with previous studies (Moral et al. 2008; Sánchez and González 2005; Abubaker et al. 2015).

The content of micronutrients and heavy metals in the slurry was not statistically affected by animal category in the case of $\mathrm{Al}, \mathrm{B}, \mathrm{Cd}, \mathrm{Co}, \mathrm{Cr}, \mathrm{Fe}, \mathrm{Li}, \mathrm{Mn}$, $\mathrm{Mo}, \mathrm{Ni}$ and $\mathrm{Pb}$. However, the content of $\mathrm{Cu}, \mathrm{Zn}$ and $\mathrm{Na}$ were affected by the animal category. Particularly, the content of $\mathrm{Cu}$ and $\mathrm{Zn}$, which are commonly used as promotors, were highest for nursery piglets (32.8 and $316.8 \mathrm{~g} \mathrm{~m}^{-3}$ respectively, which is about 3 to 6 times 
Table 3 Correlation matrix between the main pig slurry characteristics

\begin{tabular}{|c|c|c|c|c|c|c|c|c|c|c|c|c|c|c|}
\hline $\begin{array}{l}\text { Slurry } \\
\text { characteristic }\end{array}$ & $\mathrm{pH}$ & \multicolumn{2}{|l|}{$\mathrm{EC}$} & \multicolumn{2}{|l|}{$\mathrm{DM}$} & \multicolumn{2}{|l|}{ Ash } & \multicolumn{2}{|l|}{ TKN } & \multicolumn{2}{|l|}{ TAN } & $\mathrm{OM}$ & VFA & \multirow[t]{2}{*}{$\begin{array}{l}\text { Acetic } \\
\text { acid }\end{array}$} \\
\hline $\mathrm{EC}$ & $-0.069 n s$ & & & & & & & & & & & & & \\
\hline DM & $-0.068 \mathrm{~ns}$ & \multicolumn{4}{|c|}{$0.726^{* * * *}$} & & & & & & & & & \\
\hline Ash & $0.585^{* * * * *}$ & \multicolumn{2}{|c|}{$-0.362 * * *$} & \multicolumn{4}{|c|}{$-0.557 * * *$} & & & & & & & \\
\hline TKN & $-0.176 \mathrm{~ns}$ & \multicolumn{2}{|c|}{$0.816^{* * *}$} & \multicolumn{2}{|c|}{$0.867 * * *$} & \multicolumn{2}{|c|}{$-0.509 * * *$} & & & & & & & \\
\hline TAN & $-0.134 \mathrm{~ns}$ & \multicolumn{2}{|c|}{$0.888 * * *$} & \multicolumn{2}{|c|}{$0.801 * * *$} & \multicolumn{2}{|c|}{$-0.467 * * *$} & \multicolumn{2}{|c|}{$0.960 * * *$} & & & & & \\
\hline OM & $-0.097 \mathrm{~ns}$ & \multicolumn{2}{|c|}{$0.729 * * *$} & \multicolumn{2}{|c|}{$0.996 * * *$} & \multicolumn{2}{|c|}{$-0.595^{* * * * *}$} & \multicolumn{2}{|c|}{$0.872 * * *$} & \multicolumn{2}{|c|}{$0.816 * * *$} & & & \\
\hline VFA & $-0.523 * * * *$ & \multicolumn{2}{|c|}{$0.720^{* * * * *}$} & 0.489 & $* * *$ & $-0.544 *$ & $* * * *$ & 0.568 & $* * * *$ & 0.656 & $* * *$ & $0.516^{* * * * *}$ & & \\
\hline Acetic & $-0.409^{* *}$ & 0.7 & $74 * * * *$ & 0.545 & $* * *$ & $-0.507 *$ & $* * * *$ & 0.599 & $* * * *$ & 0.698 & $* * *$ & $0.569 * * * *$ & $0.957 * * *$ & \\
\hline Propionic & $-0.444 * *$ & 0.5 & $81 * * * *$ & 0.324 & & $-0.448 *$ & $* * *$ & 0.447 & $* * * *$ & 0.509 & $* * *$ & $0.340 * *$ & $0.846 * * *$ & $0.708 * * *$ \\
\hline Butyric & $-0.677 * * *$ & 0.4 & $61 * * *$ & 0.341 & & $-0.518^{*}$ & $* * *$ & 0.404 & & $0.459^{*}$ & $* * *$ & $0.376 * *$ & $0.846^{* * *}$ & $0.741 * * *$ \\
\hline $\mathrm{P}$ & $0.375^{* *}$ & -0.2 & $89 *$ & -0.085 & & $0.271 *$ & & -0.216 & & $-0.310^{*}$ & & $-0.131 \mathrm{~ns}$ & $-0.504 * * *$ & $-0.438 * * *$ \\
\hline K & $0.243 \mathrm{~ns}$ & -0.2 & $05 \mathrm{~ns}$ & -0.554 & **** & $0.659 *$ & $* * *$ & -0.342 & & $-0.237 \mathrm{r}$ & & $-0.552 * * *$ & $-0.182 \mathrm{~ns}$ & $-0.187 \mathrm{~ns}$ \\
\hline $\mathrm{Ca}$ & $0.452 * * * *$ & -0.1 & $85 \mathrm{~ns}$ & -0.078 & & $0.490 *$ & $* * * *$ & -0.161 & & -0.2511 & & $-0.127 \mathrm{~ns}$ & $-0.485 * * *$ & $-0.420^{* * * *}$ \\
\hline $\mathrm{C}$ & $-0.577 * * * *$ & 0.4 & $16^{* * * * *}$ & 0.404 & & $-0.750 \%$ & $* * * *$ & 0.475 & $* * * *$ & $0.451^{*}$ & $* * *$ & $0.426^{* * * *}$ & $0.606 * * *$ & $0.541^{* * * *}$ \\
\hline Ratio C/N & $0.430^{* * * *}$ & -0.2 & $38 \mathrm{~ns}$ & -0.014 & & $0.424 *$ & $* * *$ & -0.135 & & -0.1771 & & $-0.05 n s$ & $-0.434 * * *$ & $-0.375^{* * *}$ \\
\hline Emission $\mathrm{NH}_{3}$ & $0.176 \mathrm{~ns}$ & 0.5 & $43 * * *$ & 0.312 & & $-0.148 n$ & & 0.513 & ;**** & 0.652 & $* * *$ & $0.327^{*}$ & $0.362 * *$ & $0.420 * * *$ \\
\hline BMP & $-0.316^{*}$ & & $98 * * *$ & 0.805 & $* * *$ & $-0.587 *$ & $* * *$ & 0.896 & $* * * *$ & $0.903^{*}$ & $* * *$ & $0.836 * * *$ & $0.731 * * *$ & $0.737 * * *$ \\
\hline Slurry characteristic & Propionic a & acid & Butyri & ic acid & $\mathrm{P}$ & & $\mathrm{K}$ & & $\mathrm{Ca}$ & & $\mathrm{C}$ & Rati & tio $\mathrm{C} / \mathrm{N}$ & mission $\mathrm{NH}_{3}$ \\
\hline $\mathrm{EC}$ & & & & & & & & & & & & & & \\
\hline DM & & & & & & & & & & & & & & \\
\hline Ash & & & & & & & & & & & & & & \\
\hline TKN & & & & & & & & & & & & & & \\
\hline $\mathrm{TAN}$ & & & & & & & & & & & & & & \\
\hline OM & & & & & & & & & & & & & & \\
\hline VFA & & & & & & & & & & & & & & \\
\hline Acetic & & & & & & & & & & & & & & \\
\hline Propionic & & & & & & & & & & & & & & \\
\hline Butyric & $0.637 * * *$ & & & & & & & & & & & & & \\
\hline $\mathrm{P}$ & $-0.456^{* * *}$ & & -0.50 & $3 * * *$ & & & & & & & & & & \\
\hline K & $-0.073 \mathrm{~ns}$ & & -0.20 & $4 n s$ & & $.025 \mathrm{~ns}$ & & & & & & & & \\
\hline $\mathrm{Ca}$ & $-0.499 * * *$ & & -0.46 & $4 * * *$ & & $600 * * *$ & -0 & $.085 \mathrm{~ns}$ & & & & & & \\
\hline $\mathrm{C}$ & $0.615^{* * * *}$ & & 0.48 & $4 * * * *$ & -0 & $408^{* * *}$ & -0 & $373 * *$ & -0.4 & $470^{* * * * * * * 1 *}$ & & & & \\
\hline Ratio C/N & $-0.445^{* * * *}$ & & -0.37 & $3 * *$ & & $.383^{* * *}$ & & $156 \mathrm{~ns}$ & & $481 * * * *$ & -0.5 & $.541^{* * * *}$ & & \\
\hline Emission $\mathrm{NH}_{3}$ & $0.295^{*}$ & & 0.16 & $2 n s$ & -0 . & $.189 \mathrm{~ns}$ & & $.124 n s$ & -0.2 & $220 n s$ & & $.137 \mathrm{~ns} \quad 0$. & $.045 n s$ & \\
\hline BMP & $0.561 * * *$ & & 0.58 & $1 * * *$ & -0 . & $189 * *$ & & $.124 *$ & -0.2 & $2196^{* * *}$ & & $137 * * *-0$. & $.219 \mathrm{~ns}$ & $611^{* * * * *}$ \\
\hline
\end{tabular}

$E C$ electrical conductivity, $D M$ dry matter, $T K N$ total Kjeldahl nitrogen, $T A N$ total ammonia nitrogen, $O M$ organic matter, $V F A$ volatile fatty acids, $P$ phosphorus, $K$ potassium, $\mathrm{Ca}$ calcium, $\mathrm{C}$ carbon, $\mathrm{NH}_{3}$ ammonia, $\mathrm{BMP}$ biochemical methane potential *,**, *** Significant at $p<0.05 ; p<0.01 ; p<0.001$, respectively; $n s$ not significant

higher than other animal category; $p<0.001)$. On the contrary, the Na content was highest $(p<0.05)$ for growing animals $\left(0.53 \mathrm{~kg} \mathrm{~m}^{-3}\right)$. The content of heavy metals expressed per dry matter basis is within the range reported by Nicholson et al. (1999). These authors reported average concentrations of $\mathrm{Cu}, \mathrm{Ni}, \mathrm{Pb}$ and $\mathrm{Cd}\left(351,10.4,2.48,0.30 \mathrm{mg} \mathrm{kg}^{-1} \mathrm{DM}\right.$, respectively), which is very similar to this study $(297,8.27$, 
Fig. 1 Relationship between the electric conductivity (EC) and amount of total Kjeldahl nitrogen (TKN), total ammonia nitrogen (TAN), $\mathrm{CH}_{4}$ emission and $\mathrm{NH}_{3}$ emission in pig slurry samples $(\mathrm{n}=77)$
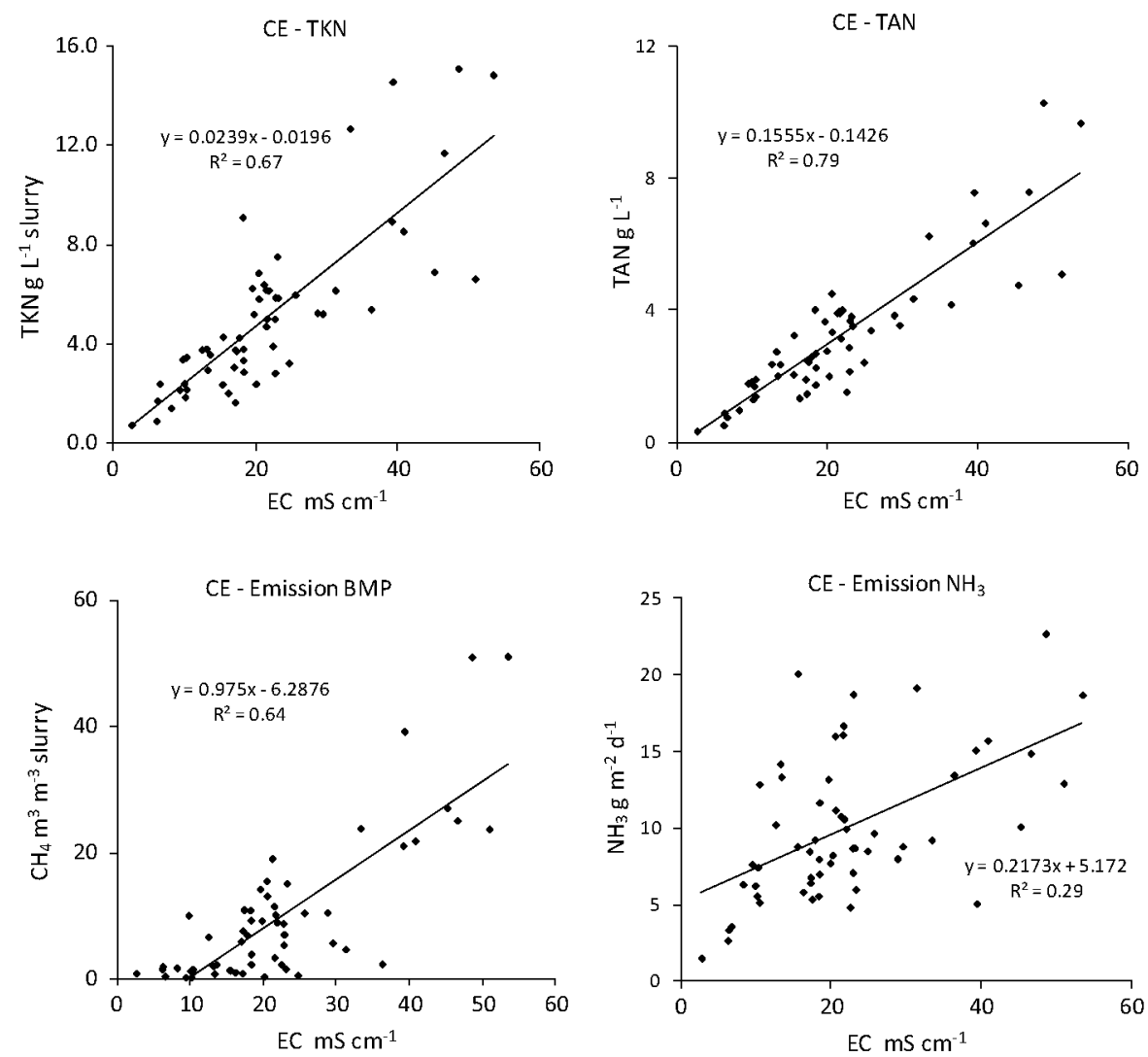

2.97 and $0.32 \mathrm{mg} \mathrm{kg}^{-1} \mathrm{DM}$, respectively). On the contrary, the concentration of $\mathrm{Zn}$ and $\mathrm{Cr}$ was from 3 to 5 times higher in our study ( 2540 vs. $575 \mathrm{mg} \mathrm{kg}^{-1} \mathrm{DM}$ for $\mathrm{Zn}$ and 10.7 vs. $2.82 \mathrm{mg} \mathrm{kg}^{-1} \mathrm{DM}$ for $\mathrm{Cr}$, respectively).

Finally, the content in VFA in the slurry was higher for growing animals than for sows $(p=0.019)$. These results are coherent with those obtained by Conn et al. (2007), who found higher nutrient and VFA contents for finishing animals and attributed these differences to the dilution of slurry. Storage time and temperature have also been reported to reduce the VFA content (Popovic and Jensen 2012; Moset et al. 2012), while a dependency between animal nutrition and the VFA content of pig slurry was reported Aarnink and Verstegen (2007).

Gaseous emissions

The measured $\mathrm{NH}_{3}$ emission of slurry was on average $10 \mathrm{~g} \mathrm{~m}^{-2}$ day $^{-1}$, ranging from 1.47 to $22.7 \mathrm{~g} \mathrm{~m}^{-2}$ day $^{-1}$ (Table 2). The emissions were higher for slurries from growing pigs $\left(11.6 \mathrm{~g} \mathrm{~m}^{-2} \mathrm{day}^{-1}\right)$, gestating sows $\left(9.37 \mathrm{~g} \mathrm{~m}^{-2} \mathrm{day}^{-1}\right)$ and nursery piglets $\left(8.43 \mathrm{~g} \mathrm{~m}^{-2}\right.$ day $\left.^{-1}\right)$, compared to lactating sows $\left(7.29 \mathrm{~g} \mathrm{~m}^{-2}\right.$ day $\left.^{-1}\right)(p=0.05)$. These results are apparently associated with the amount of TKN $(\mathbf{r}=0.51)$ and TAN $(\mathrm{r}=0.65)$ in slurries, which in turn is affected by the level of protein used in the diet (Beccaccia et al. 2015). As mentioned before, the TKN present in slurries is mainly in form of TAN (65\% in our slurry), which is the main source of $\mathrm{NH}_{3}$ emission to the atmosphere. The content of TAN in pig slurries from growing pigs and gestating sows (3.94 and $2.87 \mathrm{~kg} \mathrm{~m}^{3}$, respectively) were higher than for lactating sows and nursery piglets (1.94 and $2.32 \mathrm{~kg} \mathrm{~m}^{3}$, respectively), which would indicate a greater availability for volatilization of $\mathrm{NH}_{3}$ in slurry from growing pigs and gestating sows. A positive correlation was also found between $\mathrm{NH}_{3}$ emissions and EC, but not with slurry pH (Table 3; Fig. 1). This finding is relevant because $\mathrm{pH}$ has been mentioned as an essential factor affecting $\mathrm{NH}_{3}$ emissions (Snoek et al. 2014), but the results of this study do not reflect a 
direct effect of $\mathrm{pH}$ in a univariate analysis. Considering the variable nature of the samples from commercial farms used in this study, this suggests that the effect of $\mathrm{pH}$ may be partially confounded with the effect of TAN. Therefore, the relevance of these factors under commercial management should be further explored.

The BMP showed a wide variation, from 0.22 to $51 \mathrm{~m}^{3}$ of $\mathrm{CH}_{4}$ per $\mathrm{m}^{3}$ of slurry (Table 2). The emissions of slurries from growing pigs and nursery piglets had a significantly higher BMP (14.4 and 7.33 $\mathrm{m}^{3}$ of $\mathrm{CH}_{4}$ per $\mathrm{m}^{3}$ of slurry respectively) than those from gestating and lactating sows (4.09 and $4.45 \mathrm{~m}^{3}$ of $\mathrm{CH}_{4}$ per $\mathrm{m}^{3}$ of slurry respectively). As indicated by Gopalan et al. (2013) and Beccaccia et al. (2015), changes in management practices and nutrition may be responsible for these differences between animal categories. The BMP was positively related with EC ( $r=0.798$; Fig. 1; Table 3 ) as well as acetic, propionic, butyric acids (Table $3 ; \mathrm{r}=0.737,0.561$ and 0.581 , respectively). As indicated by Adekunle and Okolie (2015), these VFA are precursors of methanogenic activity and therefore may be potential indicators of BMP. Although accumulation of VFA may inhibit methanogenesis (Weiland 2010), in the conditions reported in this study, it seems that no inhibition was produced. The VFA content showed similar differences among animal categories as the BMP, and was higher for growing animals than for sows (Table 2). As indicated by Beccaccia et al. (2015), different animal categories are fed with different contents and types of fibers, which can influence the VFA contents in slurry.

The relationships described above can be used to predict potential emissions from slurry characteristics, as suggested by Yaguie et al. (2012) for other slurry physicochemical characteristics. Prediction equations for emission $\mathrm{CH}_{4}$ and $\mathrm{NH}_{3}$ were obtained, from easily measurable characteristics such as EC, $\mathrm{pH}$ and DM (Table 4). It can be observed that equations for predicting $\mathrm{CH}_{4}$ emissions had higher coefficients of determination than those for $\mathrm{NH}_{3}$ emissions $\left(\mathrm{R}^{2}\right.$ from 0.64 to 0.76 for $\mathrm{CH}_{4}$ and from 0.30 to 0.51 for $\mathrm{NH}_{3}$ ). However, the prediction equations improved when the models incorporated additional characteristics of the slurry (TKN, OM and TAN). Coefficients of determination including these variables reached 0.9 and 0.8 for $\mathrm{CH}_{4}$ and $\mathrm{NH}_{3}$, respectively. Other authors such as (Triolo et al. 2011) proposed prediction equations of
BMP based on the content of different fiber fractions. However, the equations presented here can be of highest interest when estimating or comparing potential emissions per volume of slurry at farm level because easily measurable variables have been considered in the prediction models.

\section{Potential fertilizer use}

Considering the maximum permissible application rate of livestock manure to lanspecified in European regulations $\left(170 \mathrm{~kg} \mathrm{~N} \mathrm{ha}^{-1}\right.$ year $^{-1}$ ), Table 5 shows nutrient inputs per hectare for land application using the slurries analyzed in this study. The volume of slurry necessary to achieve a supply of $170 \mathrm{~kg} \mathrm{~N} \mathrm{ha}^{-1}$ was on average about $52 \mathrm{~m}^{3} \mathrm{ha}^{-1}$, but was highly variable among farms (from 11 to $236 \mathrm{~m}^{3} \mathrm{ha}^{-1}$ ) because of the variation in the concentration of $\mathrm{N}$ in the slurries. The corresponding application of $\mathrm{OM}$ ranged from 259 to $3520 \mathrm{~kg} \mathrm{ha}^{-1}$. As evidenced by previous studies (Fangueiro et al. 2012b; Hernández et al. 2007), the input of OM to soil through pig slurry is relatively low compared with other manure categories due to the relatively small content of organic carbon and the labile nature of the organic compounds. Therefore, according to these authors, the application of pig slurry does not significantly contribute to soil OM but it has been suggested that in the short term it can induce a reactivation of soil microbial activity.

The amount of mineral N incorporated as TAN also varied considerably among animal categories, with different implications. As indicated by Fangueiro et al. (2012a) and evidenced in this study, pig slurry contributes high proportions of TAN, and therefore it may be a valuable fertilizer for supplying $N$ to plants. However, land application of livestock manures is associated to pollution risks related to $\mathrm{NH}_{3}$ emissions (Krupa 2003) and N leaching (Mantovi et al. 2006). As mentioned before, due to the high TAN content found in commercial slurries, there is a high potential of $\mathrm{NH}_{3}$ emission to the atmosphere. For this reason, abatement techniques during land application of pig slurry (e.g. surface application or injection) are of highest relevance (Webb et al. 2005).

The application of $\mathrm{P}$ and $\mathrm{K}$ varied by a factor 22 and 4 , respectively among slurries. The variation in $\mathrm{P}$ load was particularly relevant for gestating sows (from 5.6 to $120.6 \mathrm{~kg} \mathrm{ha}^{-1}$ ). Agriculture uses about $80 \%$ of 
Table 4 Prediction equations for BMP $\left(\mathrm{L} \mathrm{L}^{-1}\right)$ and $\mathrm{NH}_{3}$ potential emissions $\left(\mathrm{g} \mathrm{m}^{-2}\right.$ day $\left.{ }^{-1}\right)$ from pig slurry $(\mathrm{n}=77)$

\begin{tabular}{|c|c|c|c|}
\hline No. & Equations & $\mathrm{R}^{2}$ & RSD \\
\hline 1 & $\mathrm{CH}_{4}$ emission $=-6.29( \pm 0.680)+0.975( \pm 0.070) \mathrm{EC}$ & 0.637 & 4.6 \\
\hline 2 & $\mathrm{CH}_{4}$ emission $=56.18( \pm 13.13)+0.684( \pm 0.062) \mathrm{EC}-8.17( \pm 1.74) \mathrm{pH}$ & 0.658 & 4.3 \\
\hline 3 & $\mathrm{CH}_{4}$ emission $=51.61( \pm 11.17)-7.51( \pm 1.48) \mathrm{EC}+0.382( \pm 0.076) \mathrm{pH}+1.24( \pm 0.23) \mathrm{DM}$ & 0.757 & 3.5 \\
\hline 4 & $\begin{array}{l}\mathrm{CH}_{4} \text { emission }=23.79( \pm 7.93)+0.19( \pm 0.060) \mathrm{EC}-3.54( \pm 1.06) \mathrm{pH}-10.01( \pm 1.40) \mathrm{DM}+1.42 \\
( \pm 0.252) \mathrm{TKN}+13.84( \pm 1.79) \mathrm{OM}\end{array}$ & 0.896 & 2.4 \\
\hline 5 & $\mathrm{NH}_{3}$ emission $=5.17( \pm 0.919)+0.217( \pm 0.037) \mathrm{EC}$ & 0.296 & 30.6 \\
\hline 6 & $\mathrm{NH}_{3}$ emission $=-12.74( \pm 7.69)+0.223( \pm 0.036) \mathrm{EC}+2.78( \pm 1.02) \mathrm{pH}$ & 0.431 & 26.9 \\
\hline 7 & $\mathrm{NH}_{3}$ emission $=-18.48( \pm 7.12)+0.172( \pm 0.056) \mathrm{EC}+2.99( \pm 0.939) \mathrm{pH}-0.603( \pm 0.162) \mathrm{DM}$ & 0.509 & 24.6 \\
\hline 8 & $\mathrm{NH}_{3}$ emission $=-14.67( \pm 4.05)+2.47( \pm 0.529) \mathrm{pH}-0.623( \pm 0.085) \mathrm{DM}+27.12( \pm 1.81) \mathrm{TAN}$ & 0.797 & 14.4 \\
\hline
\end{tabular}

$R S D$ residual standard deviation, $E C$ electric conductivity $\mathrm{mS} \mathrm{cm}^{-1}, D M$ dry matter, in percentage per L of slurry, $O M$ organic matter, in percentage per $\mathrm{L}$ of slurry, $T K N$ total Kjeldahl nitrogen, in percentage per $\mathrm{L}$ of slurry, $T A N$ total ammonia nitrogen, in percentage per $L$ of slurry

global P flows, and an efficient use of this nutrient is essential due to its non-renewable nature and the potential environmental impacts (Schröder et al. 2010). As described by Schoumans et al. (2014) and Dourmad and Jondreville (2007) adopting feeding strategies (use of highly digestible mineral $\mathrm{P}$ supplements, use of phytase and phase feeding) may considerably change the absorption and excretion balance of the animals. Therefore, different implementation of these techniques in commercial farms may be also contributing to the wide variation in the $P$ content of pig slurry.

As indicated before, the concentration of macro nutrients expressed on a wet basis was higher for growing pigs and gestating sows than for nursery piglets and lactating sows. Furthermore, the ratio of major nutrients $\mathrm{N}: \mathrm{P}: \mathrm{K}$ in manure varied between animal categories (growing pigs 1:0.13:0.34; gestating sows 1:0.30:0.34; lactating sows 1:0.20:0.31; and nursery piglets $1: 0.16: 0.38$ ). Variable ratios of these nutrients in pig slurry have been reported in the literature, particularly those corresponding to $\mathrm{N}: \mathrm{P}$ ratios. Whereas some authors (Martínez-Suller et al. 2008; Parera i Pous et al. 2010) found relatively high contents of $\mathrm{P}$ in pig slurries $(\mathrm{N}: \mathrm{P}$ ratios 1:0.51 and 1:0.59, respectively), other authors (Abubaker et al. 2015; 31 Sánchez and González 2005) detected slurries with relatively less $\mathrm{P}$ compared to $\mathrm{N}(\mathrm{N}: \mathrm{P}$ ratios 1:0.26, in both cases). In our study, the average $\mathrm{N}: \mathrm{P}$ ratio was $1: 0.18$, which means relatively lower $\mathrm{P}$ compared to $\mathrm{N}$ than the ratios found in the literature for pig slurries. Differences in $\mathrm{N}: \mathrm{P}$ ratios among studies could be attributed to factors affecting the efficiency in the use of $\mathrm{P}$ by the animals (e.g. nutritional factors), but could be also influenced by different slurry storage and sampling strategies among studies. The $\mathrm{N}: \mathrm{K}$ ratios in the literature vary from $1: 0.3$ to 1:0.9 (Sánchez and González 2005; Martínez-Suller et al. 2008), which is consistent with the average ratio found in our study $(1: 0.3)$.

These ratios, however, do not necessarily correspond to the crop needs. For example, using the information provided by Van Duivenbooden et al. (1996) for cereals, it can be calculated that the required $\mathrm{N}: \mathrm{P}: \mathrm{K}$ ratio of fertilizer required is $1: 0.39: 1.34$. According to our results, the average slurry would provide insufficient amounts of $\mathrm{P}$ and $\mathrm{K}$. Although some particular samples were equilibrated in terms of $\mathrm{N}$ and $\mathrm{P}$, most samples (particularly those from growing pigs) would be short of $\mathrm{P}$. In all cases, for cereal production the $\mathrm{K}$ content of slurry would be insufficient to cover crop requirements. In this case, the lack of other nutrients in the slurry could be easily complemented with mineral fertilizers to obtain the optimal fertilization ratio. However, in practice fertilization ratios differ widely among crops, cropping systems and soil conditions (Penha et al. 2015) and as discussed here slurry composition is also very variable. Therefore, slurry characterization is required to avoid oversupply of nutrients and the corresponding environmental consequences, as well as to optimize the efficiency in the use of nutrients.

As regards heavy metals, loading rates were in accordance to those obtained by (Nicholson et al. 
Table 5 Nutrient inputs per hectare to the soil by an application of pig slurry equivalent to $170 \mathrm{~kg} \mathrm{~N} \mathrm{ha}^{-1}$

\begin{tabular}{|c|c|c|c|c|c|c|c|c|c|c|c|c|c|c|}
\hline \multirow[t]{2}{*}{$\begin{array}{l}\text { Amount of } \\
\text { components }\end{array}$} & \multicolumn{2}{|c|}{$\begin{array}{l}\text { Total simple } \\
\text { (n:77) }\end{array}$} & \multicolumn{3}{|c|}{ Growing pigs (n:34) } & \multicolumn{3}{|c|}{ Gestating sows (n:15) } & \multicolumn{3}{|c|}{ Lactating sows (n:14) } & \multicolumn{3}{|c|}{$\begin{array}{l}\text { Nursery piglets } \\
\text { (n:14) }\end{array}$} \\
\hline & Mean & $\mathrm{CV}$ & Mean & Min & Max & Mean & Min & Max & Mean & Min & $\operatorname{Max}$ & Mean & Min & Max \\
\hline Volumen $\left(\mathrm{m}^{3}\right)$ & 51.9 & 72.2 & 40.0 & 11.3 & 119 & 50.9 & 18.7 & 105 & 58.1 & 28.5 & 101 & 75.8 & 14.6 & 236 \\
\hline Dry matter $(\mathrm{Mg})$ & 1.71 & 51.8 & 1.64 & 0.586 & 4.49 & 1.89 & 0.453 & 4.46 & 1.69 & 0.680 & 2.75 & 1.72 & 0.935 & 2.91 \\
\hline $\begin{array}{l}\text { Organic matter } \\
(\mathrm{Mg})\end{array}$ & 1.26 & 56.1 & 1.23 & 0.362 & 3.47 & 1.38 & 0.259 & 3.52 & 1.21 & 0.387 & 2.13 & 1.24 & 0.522 & 2.23 \\
\hline Ash $(\mathrm{Mg})$ & 0.454 & 44.3 & 0.408 & 0.218 & 1.13 & 0.517 & 0.196 & 0.942 & 0.473 & 0.294 & 0.722 & 0.483 & 0.363 & 0.677 \\
\hline N (kg) & 170 & 0.0 & 170 & 170 & 170 & 170 & 170 & 170 & 170 & 170 & 170 & 170 & 170 & 170 \\
\hline TAN (Kg) & 112 & 22.7 & 114 & 54 & 152 & 119 & 75 & 157 & 107 & 69 & 152 & 106 & 72 & 136 \\
\hline Organic N (kg) & 56.9 & 39.8 & 55.3 & 17.4 & 116 & 54.1 & 13.0 & 103 & 58.4 & 17.0 & 80.7 & 62.2 & 32.6 & 97.2 \\
\hline$P(k g)$ & 30.4 & 66.0 & 24.7 & 6.50 & 66.7 & 42.1 & 5.60 & 121 & 32.3 & 8.40 & 65.9 & 29.8 & 13.50 & 55.8 \\
\hline $\mathrm{K}(\mathrm{kg})$ & 61.3 & 36.5 & 59.9 & 30.0 & 136 & 60.3 & 27.4 & 105 & 58.7 & 25.8 & 97.1 & 68.6 & 26.0 & 103 \\
\hline $\mathrm{Ca}(\mathrm{kg})$ & 43.2 & 67.2 & 33.6 & 7.90 & 95.4 & 62.2 & 10.6 & 170 & 47.6 & 15.9 & 84.5 & 41.9 & 17.5 & 74.7 \\
\hline Mg (kg) & 20.4 & 67.2 & 18.8 & 2.90 & 67.9 & 23.9 & 3.20 & 69.9 & 21.8 & 4.40 & 45.2 & 19.0 & 6.10 & 36.6 \\
\hline $\mathrm{S}(\mathrm{kg})$ & 10.9 & 48.8 & 9.64 & 3.10 & 24.70 & 10.57 & 4.00 & 20.1 & 10.2 & 6.00 & 17.9 & 15.2 & 6.00 & 25.6 \\
\hline $\mathrm{Al}(\mathrm{kg})$ & 1.62 & 84.1 & 1.32 & 0.300 & 5.80 & 1.54 & 0.100 & 3.40 & 2.49 & 0.400 & 6.00 & 1.56 & 0.200 & 4.00 \\
\hline B (g) & 62.3 & 86.5 & 52.9 & 0.000 & 200 & 53.3 & 0.000 & 100 & 64.3 & 0.000 & 100 & 92.9 & 0.000 & 100 \\
\hline $\mathrm{Cd}(\mathrm{g})$ & 0.492 & 80.4 & 0.326 & 0.100 & 1.00 & 0.480 & 0.000 & 1.10 & 0.571 & 0.100 & 1.40 & 0.829 & 0.100 & 1.80 \\
\hline Co $(\mathrm{g})$ & 27.6 & 345.7 & 13.9 & 0.500 & 147 & 45.1 & 1.70 & 560 & 59.0 & 1.50 & 622 & 10.7 & 2.30 & 39.1 \\
\hline $\mathrm{Cr}(\mathrm{g})$ & 20.9 & 96.4 & 19.1 & 2.90 & 106 & 27.3 & 1.70 & 115 & 19.7 & 3.00 & 47.3 & 19.5 & 5.10 & 42.4 \\
\hline $\mathrm{Cu}(\mathrm{g})$ & 509 & 132.3 & 299 & 79 & 1518 & 209 & 21.8 & 464 & 238 & 47.2 & 562 & 1611 & 148.6 & 3388 \\
\hline $\mathrm{Fe}(\mathrm{kg})$ & 3.14 & 90.0 & 2.33 & 0.595 & 9.57 & 2.86 & 0.270 & 6.32 & 4.64 & 0.512 & 15.2 & 3.90 & 0.666 & 13.7 \\
\hline $\operatorname{Li}(\mathrm{g})$ & 6.00 & 126.0 & 5.20 & 1.20 & 38.8 & 6.13 & 1.00 & 23.8 & 7.98 & 2.00 & 30.7 & 5.84 & 2.70 & 22.5 \\
\hline $\mathrm{Mn}(\mathrm{kg})$ & 0.795 & 70.4 & 0.736 & 0.165 & 2.34 & 0.912 & 0.110 & 3.24 & 0.706 & 0.168 & 1.43 & 0.901 & 0.267 & 2.71 \\
\hline Mo (g) & 11.2 & 72.2 & 10.4 & 2.70 & 35.7 & 8.7 & 0.80 & 23.7 & 11.9 & 2.00 & 32.4 & 15.0 & 4.80 & 38.4 \\
\hline $\mathrm{Na}(\mathrm{kg})$ & 15.4 & 36.5 & 14.7 & 5.66 & 26.9 & 15.3 & 6.40 & 25.4 & 16.2 & 4.87 & 24.8 & 16.7 & 5.56 & 27.7 \\
\hline Ni (g) & 15.1 & 78.7 & 14.4 & 4.90 & 52.2 & 18.0 & 1.40 & 70.8 & 14.2 & 4.10 & 24.9 & 14.7 & 6.70 & 36.7 \\
\hline $\mathrm{Pb}(\mathrm{g})$ & 5.27 & 131.6 & 3.11 & 0.600 & 13.6 & 4.11 & 0.200 & 14.3 & 9.50 & 0.700 & 49.0 & 7.52 & 2.10 & 30.4 \\
\hline $\mathrm{Zn}(\mathrm{kg})$ & 4.58 & 151.6 & 2.09 & 0.267 & 13.0 & 1.59 & 0.158 & 3.3 & 2.54 & 0.409 & 7.0 & 15.85 & 1.43 & 31.9 \\
\hline $\mathrm{C}(\mathrm{kg})$ & 688 & 53.9 & 687 & 211 & 1940 & 709 & 125 & 1776 & 648 & 195 & 1122 & 711 & 307 & 1245 \\
\hline
\end{tabular}

$N$ nitrogen, $T A N$ total ammonia nitrogen, $P$ phosphorus, $K$ potassium, $C a$ calcium, $M g$ magnesium, $S$ sulfur, $A l$ aluminum, $B$ boron, $\mathrm{Cd}$ cadmium, $\mathrm{Co}$ cobalt, $\mathrm{Cr}$ chromium, $\mathrm{Cu}$ copper, $\mathrm{Fe}$ iron, $\mathrm{Li}$ lithium, $\mathrm{Mn}$ manganese, $\mathrm{Mo}$ molybdenum, $\mathrm{Na}$ sodium, $\mathrm{Ni}$ nickel, $\mathrm{Pb}$ lead, $Z n$ zinc, $C$ carbon

1999). Our study also evidences that the variability found in heavy metal concentrations involves variable loading rates of these elements when slurry is applied to soils. Therefore, although evident effects of heavy metals from organic substrates on crop production may not be noticeable (Diacono and Montemurro 2010), it is likely that heavy metal accumulates in areas where pig slurry is continuously applied for years (Nicholson et al. 1999). Regarding the potential effects of heavy metal accumulation, it must be considered that they also depend on their mobility. In fact, long term studies have demonstrated the accumulation of $\mathrm{Cu}, \mathrm{Zn}$ and $\mathrm{Pb}$ in soils (Diacono and Montemurro 2010), but this did not affect soil productivity. According to these authors, soil properties such as cation exchange capacity, the presence of humic substances or the water and thermic regime are key factors affecting the mobility and thus the effects of heavy metals. The analysis of these properties is however beyond the scope of this study. 


\section{Conclusions}

The composition of pig slurries from commercial farms has a wide range of variation in the main physicochemical characteristics. Some of these characteristics (pH, N, TAN, K, Ca, Cu, Na, Zn and VFA) were significantly affected by the animal category.

Emissions of $\mathrm{NH}_{3}$ and $\mathrm{CH}_{4}$ from pig slurries of commercial farms were highly related with the physicochemical composition, and were significantly affected by the animal categories. Potential emissions could be predicted using easily measurable slurry characteristics.

The direct application of pig slurries to cropland require characterization of the slurry, due to the wide variation found in its composition. Supplementation with nutrients or slurry treatment techniques may be necessary depending on slurry composition and crop needs.

Acknowledgments This project was funded by the Spanish Ministry of Science and Innovation (AGL2011-30023) and the Valencian Government (ACOMP/2013/118). We thank the BABEL Project, Building Academic Bonds between Europe and Latin America. Erasmus Mundus Programme Action 2 for $\mathrm{PhD}$ fellowships. The translation of this paper was funded by the Universitat Politècnica de València, Spain.

\section{References}

Aarnink AJA, Verstegen MWA (2007) Nutrition, key factor to reduce environmental load from pig production. Livest Sci 109(1-3):194-203

Abubaker J, Risberg K, Jönsson E, Dahlin A S, Cederlund H, Pell M (2015) Short-term effects of biogas digestates and pig slurry application on soil microbial activity. Appl Environ Soil Sci. Article ID 658542: 1-15

Adekunle KF, Okolie JA (2015) A review of biochemical process of anaerobic digestion. Adv Biosci Biotechnol 6:205-212

Angelidaki I, Alves M, Bolzonella D, Borzacconi L, Campos JL, Guwy J, Kalyuzhnyi S, Jenicek P, Van Lier JB (2009) Defining the biomethane potential (BMP) of solid organic wastes and energy crops: a proposed protocol for batch assays. Water Sci Technol 59(5):927-934

Antezana W, Calvet S, Beccaccia A, Ferrer P, De Blas C, García-Rebollar P, Cerisuelo A (2015) Effects of nutrition on digestion efficiency and gaseous emissions from slurry in growing pigs: III. Influence of varying the dietary level of calcium soap of palm fatty acids distillate with or without orange pulp supplementation. Anim Feed Sci Technol 209:128-136

APHA (2005) Standard methods for the examination of water and wastewater. Centennial, Baltimore
Bai ZH, Qin W, Chen Q, Oenema O, Zhang FS (2014) Changes in pig production in china and their effects on nitrogen and phosphorus use and losses. Environ Sci Technol 48: 12742-12749

Beccaccia A, Ferrer P, Ibáñez MA, Estellés F, Rodríguez C, Moset V, De Blas C, Calvet S, García-rebollar P (2015) Relationships among slurry characteristics and gaseous emissions at different types of commercial spanish pig farms. Span J Agric Res 13(1):1-15

Conn KL, Topp E, Lazarovits G (2007) Factors influencing the concentration of volatile fatty acids, ammonia, and other nutrients in stored liquid pig manure. J Environ Qual 36(2):440-447

Diacono M, Montemurro F (2010) Long-term effects of organic amendments on soil fertility. A review. Agron Sustain Dev 30:401-422. doi:10.1051/agro/2009040

Díez JA, Hernaiz P, Muñoz MJ, Torre A, Vallejo A (2006) Impact of pig slurry on soil properties, water salinization, nitrate leaching and crop yield in a four-year experiment in Central Spain. Soil Use Manag 20(4):444-450

Dourmad J-Y, Jondreville C (2007) Impact of nutrition on nitrogen, phosphorus, $\mathrm{Cu}$ and $\mathrm{Zn}$ in pig manure, and on emissions of ammonia and odours. Livest Sci 112(3): 192-198

EUROSTAT (2015) Pig farming sector-statistical portrait. Pig Farming in the European Union: considerable variations from one member state to another. Statistics in Focus 15/2014. Author: Pol Marquer, Teresa Rabade, Roberta Forti ISSN:2314-9647, Catalogue number: KS-SF-14-015EN-N

Fangueiro D, Lopes C, Surgy S, Vasconcelos E (2012a) Effect of the pig slurry separation techniques on the characteristics and potential availability of $\mathrm{N}$ to plants in the resulting liquid and solid fractions. Biosyst Eng 113(2):187-194

Fangueiro D, Ribeiro H, Vasconcelos E, Coutinho J, Cabral F (2012b) Influence of animal slurries composition and relative particle size fractions on the $\mathrm{C}$ and $\mathrm{N}$ mineralization following soil incorporation. Biomass Bioenergy 47:50-51

Ferrer P, Cambra-López M, Cerisuelo A, Peñaranda D, Moset V (2014) The use of agricultural substrates to improve methane yield in anaerobic co-digestion with pig slurry: effect of substrate type and inclusion level. Waste Manag 34:196-203

Galassi G, Colombini S, Malagutti L, Crovetto GM, Rapetti L (2010) Effects of high fibre and low protein diets on performance, digestibility, nitrogen excretion and ammonia emission in the heavy pig. Anim Feed Sci Technol 161:3-4

Gopalan P, Jensen PD, Batstone DJ (2013) Anaerobic digestion of swine effluent: impact of production stages. Biomass Bioenergy 48:121-129

Hernández D, Fernández JM, Plaza C, Polo A (2007) Watersoluble organic matter and biological activity of a degraded soil amended with pig slurry. Sci Total Environ 378:101-103

Hernández D, Polo A, Plaza C (2013) Long-term effects of pig slurry on barley yield and $\mathrm{N}$ use efficiency under semiarid mediterranean conditions. Eur J Agron 44:47-86

Hristov AN, Oh J, Lee C, Meinen R, Montes F, Ott T, Firkins J, Rotz A, Dell C, Adesogan A, Yang W, Tricarico J, Kebreab E, Waghorn G, Dijkstra J, Oosting S (2013) Mitigation of greenhouse gas emissions in livestock production-A 
review of technical options for non- $\mathrm{CO}_{2}$ emissions. In: Gerber PJ, Henderson B, Makkar HPS (eds) FAO Animal Production and Health Paper No. 177. FAO, Rome

Iguácel F, Yagüe MR, Betrán J, Orús F (2011) Ensayos de Fertilización Con Purín Porcino, En Cereales de Invierno de Secano, Dirección General de Desarrollo Rural, Centro de Transferencia Agroalimentaria, Gobierno de Aragón. Informe Técnico 226:15

Jarret G, Cerisuelo A, Peu P, Martinez J, Dourmad JY (2012) Impact of pig diets with different fibre contents on the composition of excreta and their gaseous emissions and anaerobic digestion. Agric Ecosyst Environ 160:51-58

Jouany JP (1982) Volatile fatty acid and alcohol determination in digestive contents, silage juices, bacterial cultures and anaerobic fermentor contents. Sci Alimen 2:131-144

Krupa SV (2003) Effects of atmospheric ammonia $\left(\mathrm{NH}_{3}\right)$ on terrestrial vegetation: a review. Environ Pollut 124(2):179-221

Mantovi P, Fumagalli L, Beretta GP, Guermandi M (2006) Nitrate leaching through the unsaturated zone following pig slurry applications. J Hydrol 316:1-4

Martínez-Suller L, Azzellino A, Provolo G (2008) Analysis of livestock slurries from farms across Northern Italy: relationship between indicators and nutrient content. Biosyst Eng 99(4):540-552

Moral R, Moreno-Caselles J, Perez-Murcia MD, Perez-Espinosa A, Rufete B, Paredes C (2005a) Characterisation of the organic matter pool in manures. Bioresour Technol 96(2): 153-158

Moral R, Perez-Murcia MD, Perez-Espinosa A, Moreno-Caselles J, Paredes C (2005b) Estimation of nutrient values of pig slurries in Southeast Spain using easily determined properties. Waste Manag 25(7):719-725

Moral R, Perez-Murcia MD, Perez-Espinosa A, Moreno-Caselles J, Paredes C, Rufete B (2008) Salinity, organic content, micronutrients and heavy metals in pig slurries from SouthEastern Spain. Waste Manag 28(2):367-371

Morazán H, Alvarez-Rodriguez J, Seradj AR, Balcells J, Babot D (2015) Trade-offs among growth performance, nutrient digestion and carcass traits when feeding low protein and/ or high neutral-detergent fiber diets to growing-finishing pigs. Anim Feed Sci Technol 207:168-180

Moset V, Cambra-López M, Estellés F, Torres AG, Cerisuelo A (2012) Evolution of chemical composition and gas emissions from aged pig slurry during outdoor storage with and without prior solid separation. Biosyst Eng 111(1):2-10

Ndegwa PM, Vaddella VK, Hristov N, Joo HS (2009) Measuring concentrations of ammonia in ambient air or exhaust air stream using acid traps. J Environ Qual 38(2):647-653

Nicholson FA, Chambers BJ, Williams JR, Unwin RJ (1999) Heavy metal contents of livestock feeds and animal manures in England and Wales. Bioresour Technol $70(1): 23-31$

Olusegun OS (2014) Influence of NPK 15-15-15 Fertilizer and Pig Manure on Nutrient Dynamics and Production of Cowpea, Vigna unguiculata L. Walp. Am J Agric For 2(6):267

Parera i Pous J, Olivé D, Mallol Nabot C, Torrijos NC (2010) Adaptación Del Uso de La Conductividad Eléctrica (CE) Para Determinar de Forma Rápida El Contenido En Nutrientes Del Purín Porcino En Catalunya. International
Workshop on Anaerobic Digestion of Slaughterhouse Waste, pp 67-76

Patience JF, Gould SA, Koehler D, Corrigan B, Elsbernd A, Holloway CL (2015) Super-dosed phytase improves rate and efficiency of gain in nursery pigs. Anim Ind Rep AS 661:98

Penha HG, Menezes JF, Silva CA, Lopes G, Carvalho CA, Ramos SJ, Guilherme LRG (2015) Nutrient accumulation and availability and crop yields following long-term application of pig slurry in a Brazilian Cerrado soil. Nutr Cycl Agroecosyst 101(2):259-269

Popovic O, Jensen LS (2012) Storage temperature affects distribution of carbon, VFA, ammonia, phosphorus, copper and zinc in raw pig slurry and its separated liquid fraction. Water Res 46(12):3849-3858

Provolo G, Martínez-Suller L (2007) In situ determination of slurry nutrient content by electrical conductivity. Bioresour Technol 98(17):3235-3242

Sánchez M, González JL (2005) The fertilizer value of pig slurry. I. Values depending on the type of operation. Bioresour Technol 96(10):1117-1123

SAS Institute (2008) SAS/STAT Useŕs guide, v 9.3. SAS Institute Inc., Cary

Schoumans OF, Chardon WJ, Bechmann ME, Gascuel-Odoux C, Hofman G, Kronvang B, Rubæk HG, Ulén B, Dorioz JM (2014) Mitigation options to reduce phosphorus losses from the agricultural sector and improve surface water quality: a review. Sci Total Environ 468-469:1255-1266

Schröder JJ, Cordell D, Smit AL, Rosemarin A (2010) Sustainable use of phosphorus. Plant Research International Wageningen UR. Report 357

Scotford IM, Cumby TR, White RP, Carton OT, Lorenz F, Hatterman U, Provolo G (1998) Estimation of the nutrient value of agricultural slurries by measurement of physical and chemical properties. J Agric Eng Res 71(3):291-305

Snoek DJW, Johannes DS, Ogink NWM, Koerkamp PWGG (2014) Sensitivity analysis of mechanistic models for estimating ammonia emission from dairy cow urine puddles. Biosyst Eng 121:12-24

Suresh A, Choi HL, Oh DI, Moon OK (2009) Prediction of the nutrients value and biochemical characteristics of swine slurry by measurement of EC-electrical conductivity. Bioresour Technol 100:4683-4689

Thygesen O, Triolo JM, Sommer SG (2012) Indicators of physical properties and plant nutrient content of animal slurry and separated slurry. Biol Eng Trans 5(3):123-135

Triolo JM, Sommer SG, Møller HB, Weisbjerg MR, Jiang XY (2011) A new algorithm to characterize biodegradability of biomass during anaerobic digestion: influence of lignin concentration on methane production potential. Bioresour Technol 102:9395-9402

Van Duivenbooden N, de Wit CT, Van Keulen H (1996) Nitrogen, phosphorus and potassium relations in five major cereals reviewed in respect to fertilizer recommendations using simulation modelling. Fertil Res Wagening 44:37-49

Viguria M, Sanz-Cobeña A, López DM, Arriaga H, Merino P (2015) Ammonia and greenhouse gases emission from impermeable covered storage and land application of cattle slurry to bare soil. Agric Ecosyst Environ 199:261-271

Villamar CA, Canuta T, Belmonte M, Vidal G (2012) Characterization of swine wastewater by toxicity identification 
evaluation methodology (TIE). Water Air Soil Pollut 223(1):363-369

Villamar CA, Rodríguez DC, López D, Peñuela G, Vidal G (2013) Effect of the generation and physical-chemical characterization of swine and dairy cattle slurries on treatment technologies. Waste Manage Res 31(8):820-828

Villar MC, Petrikova V, Díaz-Raviña M, Carballas T (2004) Recycling of organic wastes in burnt soils: combined application of poultry manure and plant cultivation. Waste Manage 24(4):365-370

Webb J, Menzi H, Pain BF, Misselbrook TH, Dämmgen U, Hendriks H, Döhler H (2005) Managing ammonia emissions from livestock production in Europe. Environ Pollut $135: 399-406$
Webb J, Broomfield M, Jones S, Donovan B (2014) Ammonia and odour emissions from UK pig farms and nitrogen leaching from outdoor pig production. Sci Total Environ 470:865-875

Weiland P (2010) Biogas production: current state and perspectives. Appl Microbiol Biotechnol 85:849-860

Yagüe MR, Bosch-Serra AD, Boixadera J (2012) Measurement and estimation of the fertiliser value of pig slurry by physicochemical models: usefulness and constraints. Biosyst Eng 111(2):206-216

Zhang W, Wei Q, Wu S, Qi D, Li W, Zuo Z, Dong R (2014) Batch anaerobic co-digestion of pig manure with dewatered sewage sludge under mesophilic conditions. Appl Energy 128:175-183 